\title{
Correlations Between Sociability and Motor Performance Scores in 8-Month-Olds*
}

\author{
Michael E. Lamb \\ Department of Psychology \\ University of Utah \\ Salt Lake City, UT 84112 \\ Stanley M. Garn \& Mary T. Keating \\ Center for Human Growth and Development \\ University of Michigan \\ Ann Arbor, MI 48109
}

\begin{abstract}
Correlations between nieasures of social responsiveness (to examiner and to mother), social intensity, social class, and motor development were computed using data from the eightmonth assessments of 33,688 participants in the National Collaborative Perinatal Project. A composite measure of sociability correlated .18 with motor performance scores. Of the component scales, social responsiveness to the examiner was the best correlate $(r=.19)$, followed by social intensity $(r=.09)$. There were no effects attributable to either sex or race. Social class was correlated with motor performance scores $(r=.07)$ but not with the sociability scales. Since sociability predicts motor test performance almost as well as it predicts mental development scores, if appears that a performance artifact does not account for these correlations. Direct relationships among sociability, cognitive, and motor development appear to be involved.
\end{abstract}

Within the last decade, several researchers have confirmed an early finding by Bayley and Schaefer (1964) that infants' performance on standardized tests is correlated with measures of the infants' sociability (see Lamb, 1982, for a review). The repeated demonstration of an association between sociability and cognitive test performance has led to speculation about the nature of this relationship. On the one hand, a performance effect may be involved, with sociable,

* The National Collaborative Perinatal Project was supported and coordinated by the National Institute for Neurological and Communicable Diseases and Stroke (NINCDS). The analyses reported here were funded by the Center for Human Growth and Development of the University of Michigan. We are grateful to Steve Ridella for assistance with the analyses. Correspondence and reprint requests should be addressed to Michael E. Lamb, Department of Psychology, University of Utah, Salt Lake City, UT 84112. 
cooperative babies performing better in test situations than shy or uncooperative infants. Alternately, there may be a real difference in cognitive competence attributable to the fact that sociable babies have elicited more attention and stimulation from parents and other adults than less sociable infants have. Lamb, Garn, and Keating (1981) found that sociability was slightly more highly correlated $(r=.26)$ with performance on socially-loaded (verbal and play) items than on nonsocial items $(r=.17)$, suggesting that there was a modest performance effect, as well as a clear competence effect.

All previous research has involved performance on tests of mental or cognitive development. In the present study, we sought to investigate this issue further by examining the relationship between sociability and scores on the Bayley Motor Scales. Examination of the items suggested that motor test performance should be less susceptible to performance effects than mental test performance is. Consequently, if we found correlations between sociability and motor test performance that were equivalent in magnitude to those between sociability and mental test performance, it would suggest that performance effects were not involved. Our study involved reanalyses of the same large national sample employed by Lamb et al. (1981) making comparisons between the two studies quite straightforward. They reported a correlation of .23 between a composite sociability score and total mental scale scores.

The subjects in this study were a subset of the participants in the National Collaborative Perinatal Project (NCPP) conducted by the National Institute for Neurological and Communicable Diseases and Stroke (NINCDS). All infants in this project were born between January 1959 and December 1965. Further details concerning the NCPP participants were provided in a volume by Niswander and Gordon (1972). Data were available for 8,307 Black females, 8,124 Black males, 7,432 White females, 7,635 White males, 1,110 Puerto Rican males, and 1,080 Puerto Rican females.

All the subjects were tested using a "research version" of the Bayley Motor Scale. This version did not include two items contained in the 1969 Manual: item 25 (Attempts to secure pellet) and item 26 (Rotates wrist). The infants were tested by one of a variety of examiners employed at each of the participating sites. All examiners had been thoroughly trained but no data regarding reliability were collected. All analyses reported below involved total performance scores, reflecting the number of items successfully passed.

Sociability was assessed using three of the eight rating scales completed by the examiner at the end of the test session. On the first of these scales (social responsiveness to examiner), a score of 1 indicated "Avoids, draws back, turns to mother"; 2 implied "Hesitates, is apprehensive of approach of examiner"; 3- "Accepts, is passive, but responds"; 4- "Friendly, responds easily to most test situations"; and 5- "Invites, instigates social contacts." On the second scale (social responsiveness to mother), 1 was scored when the infant "Ignores mother during free play, resists contact with mother"; 2-“Hesitates, cooperates 
in certain tests"; 3- "Accepts, responds adequately to assistance by mother"; 4 "Enjoys contact with mother during testing"; and 5-_ "Demands, clings to mother." The third rating scale measured intensity of social responses. On this scale, 1 meant "Does not respond to initiation of social contact"; 2- "Responds only to direct approach, no interest in persons"; 3- "As interested in social contact as in object manipulation"; 4- "Behavior strongly affected by persons, more interested in persons than objects"; and 5- "Very strong-over-reacts to persons." Scores on these scales were also combined into a composite sociability score. The other five rating scales were not measures of social behavior (activity level, speed, intensity, duration, and persistence of responses).

Social class was assessed using the PRB index which was developed for use in the Collaborative Project (Myrianthopoulos \& French, 1968). The index considers parental income, education, and occupation, with possible scores ranging from 0 to 95 .

Because of the sample size, it was not possible to analyze the complete data set, so data for males and females were analyzed separately. The correlations between motor performance and composite sociability scores were .18 for males and .17 for females $(p$ 's $<.00001)$. The highest correlations were with social responsiveness to the examiner ( $r$ 's $=.19, p$ 's $<.00001)$; correlations between motor scores and social responsiveness to mother were .08 (males) and .06 (females) $(p$ 's $\dot{<} .00001)$, and between motor scales and social intensity were .10 and .09 ( $p$ 's $<.00001)$ respectively. Motor scores were also correlated with social class ( $r$ 's $=.08$ and $.05, p$ 's $<.00001$, respectively). The correlations for males and females were not significantly different. There were no significant effects on either raw scores or correlations attributable to race.

Stepwise multiple regression equations were computed to determine whether the three measures of sociability and the social class index were independently correlated with the motor scores. Computer limitations made it necessary to compute these regressions separately for each race by sex subgroup, but the results were similar in every case. Social-responsiveness to the examiner was always the first predictor to enter, with partial correlations ranging from .16 to $.23 \overline{(X}=.20, p$ 's $<.00001)$. The second predictor was always social class, with partial correlations ranging from .06 to $.10(\bar{X}=.09, p$ 's $<.002)$ followed by social intensity, with partial correlations ranging from .04 to $.08(\bar{X}=.06, p$ 's $<$ $.009)$. Thus, these three factors contributed independently to variations in the motor scales. Further details concerning the multiple regression equations are available from the first author.

These analyses demonstrate quite convincingly that infant sociability is a significant correlate of motor performance scores, just as it is a reliable predictor of mental performance scales (Lamb, 1982). Social responsiveness to the examiner was the strongest predictor of the motor performance scores; correlations between this variable and the motor scores were about the same as the correlations between the composite sociability and the motor scale scores. 
The consistent correlations between measures of sociability and motor performance suggest that sociability affects performance via an effect on competence rather than via a performance artifact, since it is unlikely that the sociability of the infant during a test session could affect motor as much as mental task performance. This interpretation is supported by a comparison with the data presented by Lamb et al. (1981). The correlations they reported between mental test scores and social responsiveness to the examiner are only marginally greater than the correlations we found between motor performance and social responsiveness to the examiner (.23 vs .19). It is implausible than the infant's responsiveness to the examiner should directly affect estimates of its motor skills in the same way that it might affect estimates of its mental capacity. However, we cannot exclude the possibility of a halo effect, since the ratings were made by the testers. Future studies must be much more careful to ensure independent assessments. Further, the conclusion that sociability affects motor and mental competence directly-presumably by affecting the amount of formative stimulation and attention children receive-is by no means proven by the correlations obtained in the research conducted thus far. It could be that social, cognitive, and motor competence are intercorrelated aspects of development such that more sociable infants are also more advanced cognitively and motorically. Longitudinal studies tracing the relationships among these variables over time are called for.

The correlations reported here, while highly reliable, are extremely low. While they are comparable to those obtained by Lamb et al. (1981) in an earlier analysis of the Collaborative Project data, they are much lower than those obtained in studies involving smaller samples (see Lamb, 1982). This is probably because the measures of sociability used in the Collaborative Project were less discriminating, and had a restricted range. For example, the published Bayley (1969) manual contains three 9-point indices of sociability with the examiner (all of which were used by researchers such as Stevenson \& Lamb, 1979) compared with one 5-point scale. The measures of social responsiveness to mother and social intensity do not clearly tap sociability as defined by Lamb (1982); and the index of social responsiveness to mother is not a linear ordinal scale. All of these factors may have attenuated the magnitude of the correlations obtained. Nevertheless, it seems that a reliable phenomenon exists: further research is needed to determine its magnitude and psychological importance.

\section{REFERENCES}

Bayley, N. Bayley scales of infant development. New York: Psychological Corporation, 1969.

Bayley, N., \& Schaefer, E. S. Correlations of maternal and child behaviors with the development of mental abilities: Data from the Berkeley Growth Study. Monographs of the Society for Research in Child Development, 1964, 29 (6, Whole No. 97).

Lamb, M. E. The origins of individual differences in infant sociability and their implications for 
cognitive development. In H. W. Reese \& L. P. Lipsitt (Edš.), Advances in child development and behavior (Vol. 16). New York: Academic, 1982.

Lamb, M. E., Gam, S. M., \& Keating, M. T. Correlations between sociability and cognitive performance among 8-month-olds. Child Development, 1981, 52, 711-713.

Myrianthopoulos, N. C., \& French, K. S. An application of the U. S. Bureau of the Census Socioeconomic Index to a large, diversified patient population. Social Science and Medicine, 1968, 2, 283-299:

Niswander, K. R., \& Gordon, M. The Collaborative Perinatal Study of the National Institute of Neurological Diseases and Stroke: The women and their pregnancies. Philadelphia: Saunders, 1972.

Stevenson, M. B., \& Lamb, M. E. Effects of infant sociability and the caretaking environment on infant cognitive performance. Child Development, 1979, 50, 340-349. 